just seen, its coefficients are rational. It follows from the definition that the equation ( 1 ) is reducible.

Conversely, it is now easy to see that if the equation (1) is reducible, its group must be intransitive.

We are therefore enabled to enunciate the following theorem, exactly analogous to a theorem in algebra :

The group of an irreducible linear differential equation is transitive.

GöTTINGEN, INecember, 1901.

\title{
LINES OF LENGTH ZERO ON SURFACES.
}

BY DR. L. P. EISENHART.

(Read before the American Mathematical Society, December 28, 1901.)

By definition, the directions of the double system of lines of length zero on a surface are given by equating to zero the expression for the square of the linear element of the given surface, that is, in the Gauss notation,

$$
E d u^{2}+2 F d u d v+G d v^{2}=0 .
$$

Since these lines are always imaginary and since the lines of curvature of a real surface are always real there is no real surface whose lines of curvature are of length zero. It is well known that lines of length zero form a conjugate system upon a minimal surface and further that this is a characteristic property of such surfaces. In order that asymptotic lines be of length zero it is necessary and sufficient that the two fundamental forms of the surface be proportional. Hence the sphere is the only real surface whose asymptotic lines are of length zero ; they are the imaginary rectilinear generatrices of the sphere. When the parameters of these lines are taken as conjugate imaginaries and the sphere is of radius unity and with center at the origin, the cartesian coördinates have the following expressions :

$$
x=\frac{u+v}{1+u v}, \quad y=i \frac{v-u}{1+u v}, \quad z=\frac{u v-1}{1+u v} . *
$$

We propose now the following problem :

\footnotetext{
* Darboux, Leçons, I, p. 245.
} 
For what surfaces is the spherical representation of lines of length zero on the surface given by lines of length zero on the sphere?

When $u=$ const., $v=$ const. on the sphere are lines of length zero, the formulæ (2) give the expressions for the direction cosines of the normal to the surface $S$, hence the surface is the envelope of the plane whose equation is

$$
(u+v) x+i(v-u) y+(u v-1) z+\xi=0
$$

and the cartesian coördinates of points on $S$ are given by*

where

$$
\begin{gathered}
x+i y=-v z-p, \quad x-i y=-u z-q, \\
z=\frac{\xi-p u-q v}{1+u v},
\end{gathered}
$$

$$
p, q=\frac{\partial \xi}{\partial u}, \quad \frac{\partial \xi}{\partial v}
$$

The square of the linear element of $S$ is

or, on writing

$$
d s^{2}=(z d u+d q)(z d v+d p),
$$

$$
\begin{gathered}
r, s, t=\frac{\partial^{2} \xi}{\partial u^{2}}, \quad \frac{\partial^{2} \xi}{\partial u \partial v}, \\
\partial^{2} \xi \\
\partial s^{2}=[(z+s) d u+t d v][r d u+(z+s) d v] .
\end{gathered}
$$

From this it follows that the necessary and sufficient condition that the lines $u=$ const., $v=$ const. on $S$ be of length zero is

hence either

$$
(z+s) r=0, \quad(z+s) t=0
$$

$$
z+s=0, \quad \text { or } \quad r=t=0 .
$$

The former is a characteristic equation of minimal surfaces. $\dagger$ To discuss the latter we make use of the formulæ:

$$
\begin{aligned}
& 2 \rho_{1}=\xi-p u-q v+(1+u v)(s+\sqrt{r t}), \\
& 2 \rho_{2}=\xi-p u-q v+(1+u v)(s-\sqrt{r t}),
\end{aligned}
$$

* Dauboux. leçons, I, p. 246.

† Ib., p. 297.

$\ddagger$ lb., p. $: 46$. 
where $\rho_{1}$ and $\rho_{2}$ denote the principal radii of $S$. For the second of the conditions (5) this gives $\rho_{1}=\rho_{2}$, that is, $S$ is a sphere, when real. Combining these results we have that minimal surfaces and spheres are the only real surfaces for which the spherical representation of lines of length zero is the system of rectilinear generatrices of the sphere.

In order to determine what surfaces have the lines of length zero on the sphere for spherical representation of its asymptotic lines we note that for the parameters used previously the equation of the asymptotic directions is*

$$
r d u^{2}+2(s+z) d u d v+t d v^{2}=0 .
$$

Hence, in order that the $u$ and $v$ lines may be asymptotic for the surface, the condition is

$$
r=t=0,
$$

which as we have seen characterizes the sphere. Again, in order that the parametric lines may form a conjugate system we get from the above equation the condition

$$
s+z=0,
$$

that is minimal surfaces. Recalling the above theorem we have the following:

In order that the asymptotic lines or a conjugate system on a surface may be represented upon the sphere by its imaginary generatrices, they must be lines of length zero on the surface.

Princeton,

November, 1901.

\section{SOME PROPERTIES OF POTENTIAL SURFACES.}

BY DR. EDWARD KASNER.

(Read before the American Mathematical Society, April 27, 1901.)

In a previous paper, published in the Bulletin, volume 7, pages 392-399, the author studied the algebraic curves $\varphi(x y)=0$ defined by the condition $\varphi_{x x}+\varphi_{y y} \equiv 0$. Two classes of characteristic properties were obtained, the first translating directly the differential equation and the second arising from the well-known connection between harmonic

\footnotetext{
* Darboux, Leçons, I, p. 246.
} 